\title{
Some properties of pre-quasi norm on Orlicz sequence space
}

\author{
Awad A. Bakery ${ }^{1,2^{*}}$ and Afaf R. Abou Elmatty ${ }^{2}$
}

\section{"Correspondence:}

awad_bakery@yahoo.com

'Department of Mathematics, College of Science and Arts at Khulis, University of Jeddah, Jeddah, Saudi Arabia

2Department of Mathematics, Faculty of Science, Ain Shams University, Cairo, Egypt

\begin{abstract}
In this article, we introduce the concept of pre-quasi norm on $E$ (Orlicz sequence space), which is more general than the usual norm, and give the conditions on $E$ equipped with the pre-quasi norm to be Banach space. We give the necessity and sufficient conditions on $E$ equipped with the pre-quasi norm such that the multiplication operator defined on $E$ is a bounded, approximable, invertible, Fredholm, and closed range operator. The components of pre-quasi operator ideal formed by the sequence of $s$-numbers and $E$ is strictly contained for different Orlicz functions are determined. Furthermore, we give the sufficient conditions on $E$ equipped with a pre-modular such that the pre-quasi Banach operator ideal constructed by s-numbers and $E$ is simple and its components are closed. Finally the pre-quasi operator ideal formed by the sequence of $s$-numbers and $E$ is strictly contained in the class of all bounded linear operators, whose sequence of eigenvalues belongs to $E$.
\end{abstract}

Keywords: Pre-quasi norm; Orlicz sequence space; Multiplication operator; Fredholm operator; Approximable operator; Simple Banach space

\section{Introduction}

Throughout the paper, we denote the space of all bounded linear operators from a Banach space $X$ into a Banach space $Y$ by $L(X, Y)$, and if $X=Y$, we write $L(X)$, the space of all real sequences is denoted by $w$, the real numbers $\mathbb{R}$, the complex numbers $\mathbb{C}, \mathbb{N}=\{0,1,2, \ldots\}$, the space of null sequences by $C_{0}$, and the space of bounded sequences by $\ell_{\infty}$. In operator theory, the multiplication operators on $L_{p}$-spaces are related to the composition operators; this means that the properties of composition operators on $L_{p}$-spaces can be stated by the properties of multiplication operators. Singh and Kumar [28] proved that a composition operator on $L_{p}(X ; \mathbb{C})$ is compact if and only if the multiplication operator $T_{\alpha}$ is compact, where $\alpha=\frac{d \mu T^{-1}}{d \mu}$ is the Radon-Nikodym derivative of the measure $\mu T^{-1}$ with respect to the measure $\mu$. In the theory of Hilbert space, every normal operator on a separable Hilbert space is unitarily equivalent to a multiplication operator. In the spectral theory, multiplication operators have their roots in the spectral theory. For more details on multiplication operators, see [1, 26, 27, 29-31]. On sequence spaces, Mursaleen and Noman in $[17,18]$ studied the compact operators on some difference sequence spaces; Komal and Gupta [10] studied the multiplication operators on Orlicz spaces equipped

(c) The Author(s) 2020. This article is licensed under a Creative Commons Attribution 4.0 International License, which permits use, sharing, adaptation, distribution and reproduction in any medium or format, as long as you give appropriate credit to the original author(s) and the source, provide a link to the Creative Commons licence, and indicate if changes were made. The images or other third party material in this article are included in the article's Creative Commons licence, unless indicated otherwise in a credit line to the material. If material is not included in the article's Creative Commons licence and your intended use is not permitted by statutory regulation or exceeds the permitted use, you will need to obtain permission directly from the copyright holder. To view a copy of this licence, visit http://creativecommons.org/licenses/by/4.0/. 
with the Luxemburg norm, and Komal et al. [11] examined the multiplication operators on Cesáro sequence spaces. The theory of operator ideal goals possesses an uncommon essentialness in useful examination. Some of operator ideals in the class of Banach spaces or Hilbert spaces are defined by different scalar sequence spaces. For example the ideal of compact operators is defined by the space $C_{0}$ of null sequence and Kolmogorov numbers. Pietsch [24] examined the quasi-ideals formed by the approximation numbers and classical sequence space $\ell^{p}(0<p<\infty)$. He proved that the ideals of nuclear operators and of Hilbert-Schmidt operators between Hilbert spaces are defined by $\ell^{1}$ and $\ell^{2}$, respectively. He proved that the class of all finite rank operators is dense in the Banach quasi-ideal, and the algebra $L\left(\ell^{p}\right)$, where $(1 \leq p<\infty)$, contains one and only one nontrivial closed ideal. Pietsch [23] showed that the quasi Banach operator ideal formed by the sequence of approximation numbers is small. Makarov and Faried [14] proved that the quasi-operator ideal formed by the sequence of approximation numbers is strictly contained for different powers, i.e., for any infinite dimensional Banach spaces $X, Y$ and for any $q>p>0$, it is true that $S_{\ell p}^{\text {app }}(X, Y) \varsubsetneqq S_{\ell q}^{\text {app }}(X, Y) \varsubsetneqq L(X, Y)$. In [8], Faried and Bakery studied the operator ideals constructed by approximation numbers, generalized Cesáro and Orlicz sequence spaces $\ell_{M}$. In [9], Faried and Bakery introduced the concept of pre-quasi operator ideal, which is more general than the usual classes of operator ideals. They studied the operator ideals constructed by $s$-numbers, generalized Cesáro and Orlicz sequence spaces $\ell_{M}$, and proved that the operator ideal formed by the previous sequence spaces and approximation numbers is small under certain conditions. The aim of this paper to study the concept of pre-quasi norm on $E$ (Orlicz sequence space), which is more general than the usual norm, and give the conditions for $E$ equipped with the pre-quasi norm to be Banach space. We give the necessity and sufficient conditions on $E$ equipped with the pre-quasi norm such that the multiplication operator defined on $E$ is a bounded, approximable, invertible, Fredholm, and closed range operator. The components of pre-quasi operator ideal formed by the sequence of $s$-numbers and $E$ is strictly contained for different Orlicz functions are determined. Furthermore, we give the sufficient conditions on $E$ equipped with a premodular such that the pre-quasi Banach operator ideal constructed by $s$-numbers and $E$ is simple and its components are closed. Finally the pre-quasi operator ideal formed by the sequence of $s$-numbers and $E$ is strictly contained in the class of all bounded linear operators, whose sequence of eigenvalues belongs to $E$.

\section{Definitions and preliminaries}

Definition 2.1 ([24]) A finite rank operator is a bounded linear operator whose dimension of the range space is finite. The space of all finite rank operators on $E$ is denoted by $F(E)$.

Definition 2.2 ([24]) A bounded linear operator $A: E \rightarrow E$ (where $E$ is a Banach space) is called approximable if there are $S_{n} \in F(E)$ for all $n \in \mathbb{N}$ such that $\lim _{n \rightarrow \infty}\left\|A-S_{n}\right\|=0$. The space of all approximable operators on $E$ is denoted by $\Psi(E)$, and the space of all approximable operators from $E$ to $F$ is denoted by $\Psi(E, F)$.

Lemma 2.3 ([24]) Let $T \in L(X, Y)$. If $T$ is not approximable, then there are operators $G \in$ $L(X, X)$ and $B \in L(Y, Y)$ such that $B T G e_{k}=e_{k}$ for all $k \in \mathbb{N}$.

Definition 2.4 ([24]) A Banach space $E$ is called simple if the algebra $L(E)$ contains one and only one nontrivial closed ideal. 
Definition 2.5 ([24]) A bounded linear operator $A: E \rightarrow E$ (where $E$ is a Banach space) is called compact if $A\left(B_{1}\right)$ has compact closure, where $B_{1}$ denotes the closed unit ball of $E$. The space of all compact operators on $E$ is denoted by $L_{c}(E)$.

Theorem 2.6 ([24]) If $E$ is infinite dimensional Banach space, we have

$$
F(E) \varsubsetneqq \Psi(E) \varsubsetneqq L_{c}(E) \varsubsetneqq L(E) .
$$

Definition 2.7 ([16]) A bounded linear operator $A: E \rightarrow E$ is called Fredholm if $A$ has closed range, $\operatorname{dim}(\operatorname{ker} A)$ and $\operatorname{co-dim}(\operatorname{range} A)$ are finite.

Definition 2.8 ([12]) An Orlicz function is a function $M:[0, \infty) \rightarrow[0, \infty)$, which is convex, continuous, and nondecreasing with $M(0)=0, M(u)>0$ for $u>0$ and $M(u) \rightarrow \infty$, as $u \rightarrow \infty$.

Definition 2.9 ([22]) An Orlicz function $M$ is said to satisfy $\Delta_{2}$-condition for all values of $x \geq 0$, if there exists a constant $k>0$ such that $M(2 x) \leq k M(x)$. The $\Delta_{2}$-condition is equivalent to $M(l x) \leq k l M(x)$ for all values of $x$ and for $l>1$.

Lindenstrauss and Tzafriri [13] utilized the idea of an Olicz function to define Orlicz sequence space:

$$
\ell_{M}=\{u \in \omega: \rho(\beta u)<\infty \text { for some } \beta>0\} \quad \text { where } \rho(u)=\sum_{k=0}^{\infty} M\left(\left|u_{k}\right|\right),
$$

$\left(\ell_{M},\|\cdot\|\right)$ is a Banach space with the Luxemburg norm:

$$
\|u\|=\inf \left\{\beta>0: \rho\left(\frac{u}{\beta}\right) \leq 1\right\} .
$$

Every Orlicz sequence space contains a subspace that is isomorphic to $c_{0}$ or $\ell^{q}$ for some $1 \leq q<\infty$.

As of late, different classes of sequences have been studied using Orlicz functions by Et et al. [7], Mursaleen et al. [19-21], Alotaibi et al. [2-4], and Mohiuddine et al. [15].

Definition 2.10 ([6]) A class of linear sequence spaces $\mathbb{E}$ is called a special space of sequences (sss) if

(1) $e_{i} \in \mathbb{E}$ for all $i \in \mathbb{N}$;

(2) if $u=\left(u_{i}\right) \in w, v=\left(v_{i}\right) \in \mathbb{E}$, and $\left|u_{i}\right| \leq\left|v_{i}\right|$ for every $i \in \mathbb{N}$, then $u \in \mathbb{E}$, "i.e., $\mathbb{E}$ is solid";

(3) if $\left(u_{i}\right)_{i=0}^{\infty} \in \mathbb{E}$, then $\left(u_{\left[\frac{i}{2}\right]}\right]_{i=0}^{\infty} \in \mathbb{E}$, wherever $\left[\frac{i}{2}\right]$ means the integral part of $\frac{i}{2}$.

Theorem 2.11 ([9]) $\ell_{M}$ is a (sss) if $M$ is an Orlicz function satisfying $\Delta_{2}$-condition.

Definition 2.12 ([6]) A subclass of the special space of sequences is called a pre-modular (sss) if there is a function $\varrho: \mathbb{E} \rightarrow[0, \infty[$ satisfying the following conditions:

(i) $\varrho(u) \geq 0$ for each $u \in \mathbb{E}$ and $\varrho(u)=0 \Leftrightarrow u=\theta$, where $\theta$ is the zero element of $\mathbb{E}$;

(ii) There exists $L \geq 1$ such that $\varrho(\beta u) \leq L|\beta| \varrho(u)$ for all $u \in \mathbb{E}$ and for any scalar $\beta$;

(iii) For some $K \geq 1, \varrho(u+v) \leq K(\varrho(u)+\varrho(v))$ for every $u, v \in \mathbb{E}$; 
(iv) If $\left|u_{i}\right| \leq\left|v_{i}\right|$ for all $i \in \mathbb{N}$, then $\varrho\left(\left(u_{i}\right)\right) \leq \varrho\left(\left(v_{i}\right)\right)$;

(v) For some $K_{0} \geq 1, \varrho\left(\left(u_{i}\right)\right) \leq \varrho\left(\left(u_{\left[\frac{i}{2}\right]}\right)\right) \leq K_{0} \varrho\left(\left(u_{i}\right)\right)$;

(vi) The set of all finite sequences is $\varrho$-dense in $\mathbb{E}$. This means that, for each $u=\left(u_{i}\right)_{i=o}^{\infty} \in \mathbb{E}$ and for each $\varepsilon>0$, there exists $s \in \mathbb{N}$ such that $\varrho\left(\left(u_{i}\right)_{i=s}^{\infty}\right)<\varepsilon$;

(vii) There exists a constant $\xi>0$ such that $\varrho(\beta, 0,0,0, \ldots) \geq \xi|\beta| \varrho(1,0,0,0, \ldots)$ for any $\beta \in \mathbb{R}$.

Theorem 2.13 ([9]) $\ell_{M}$ is a pre-modular (sss) if $M$ is an Orlicz function satisfying $\Delta_{2}$ condition.

Definition 2.14 ([24]) Let $L$ be a class of all bounded linear operators between any arbitrary Banach spaces. A sub class $U$ of $L$ is called an operator ideal if each element $U(X, Y)=U \cap L(X, Y)$ fulfills the following conditions:

(i) $I_{\digamma} \in U$ wherever $\digamma$ represents a Banach space of one dimension.

(ii) The space $U(X, Y)$ is linear over $\mathbb{R}$.

(iii) If $T \in L\left(X_{0}, X\right), V \in U(X, Y)$, and $R \in L\left(Y, Y_{0}\right)$, then $R V T \in U\left(X_{0}, Y_{0}\right)$.

Closed ideal means an ideal which contains its limit points.

The concept of pre-quasi operator ideal is more general than the usual classes of operator ideals.

Definition 2.15 ([6]) A function $g: \Omega \rightarrow[0, \infty)$ is said to be a pre-quasi norm on the ideal $\Omega$ if the following conditions hold:

(1) For all $T \in \Omega(X, Y), g(T) \geq 0$ and $g(T)=0$ if and only if $T=0$;

(2) There exists a constant $M \geq 1$ such that $g(\lambda T) \leq M|\lambda| g(T)$ for all $T \in \Omega(X, Y)$ and $\lambda \in \mathbb{R}$

(3) There exists a constant $K \geq 1$ such that $g\left(T_{1}+T_{2}\right) \leq K\left[g\left(T_{1}\right)+g\left(T_{2}\right)\right]$ for all $T_{1}, T_{2} \in \Omega(X, Y)$

(4) There exists a constant $C \geq 1$ such that if $T \in L\left(X_{0}, X\right), P \in \Omega(X, Y)$, and $R \in L\left(Y, Y_{0}\right)$, then $g(R P T) \leq C\|R\| g(P)\|T\|$, where $X_{0}$ and $Y_{0}$ are normed spaces.

Theorem $2.16([9])$ The function $g(P)=\varrho\left(s_{i}(P)\right)_{i=0}^{\infty}$ is a pre-quasi norm on $S_{\mathbb{E}_{\varrho}}$, where $\mathbb{E}_{\varrho}$ is a pre-modular (sss).

Definition 2.17 ([25]) An $s$-number function is a map defined on $L(X, Y)$ which associates with each operator $T \in L(X, Y)$ a nonnegative scaler sequence $\left(s_{n}(T)\right)_{n=0}^{\infty}$ assuming that the taking after states are verified:

(a) $\|T\|=s_{0}(T) \geq s_{1}(T) \geq s_{2}(T) \geq \cdots \geq 0$ for $T \in L(X, Y)$;

(b) $s_{m+n-1}\left(T_{1}+T_{2}\right) \leq s_{m}\left(T_{1}\right)+s_{n}\left(T_{2}\right)$ for all $T_{1}, T_{2} \in L(X, Y), m, n \in \mathbb{N}$;

(c) Ideal property: $s_{n}(R V T) \leq\|R\| s_{n}(V)\|T\|$ for all $T \in L\left(X_{0}, X\right), V \in L(X, Y)$, and $R \in L\left(Y, Y_{0}\right)$, where $X_{0}$ and $Y_{0}$ are arbitrary Banach spaces;

(d) If $G \in L(X, Y)$ and $\lambda \in \mathbb{R}$, we obtain $s_{n}(\lambda G)=|\lambda| s_{n}(G)$;

(e) Rank property: If $\operatorname{rank}(T) \leq n$, then $s_{n}(T)=0$ for each $T \in L(X, Y)$;

(f) Norming property: $s_{r \geq n}\left(I_{n}\right)=0$ or $s_{r<n}\left(I_{n}\right)=1$, where $I_{n}$ represents the unit operator on the $n$-dimensional Hilbert space $\ell_{2}^{n}$. 
Notations $2.18([9])$

$$
\begin{aligned}
& S_{E}:=\left\{S_{E}(X, Y) ; X \text { and } Y \text { are Banach spaces }\right\}, \text { where } \\
& S_{E}(X, Y):=\left\{T \in L(X, Y):\left(\left(s_{i}(T)\right)_{i=0}^{\infty} \in E\right\} .\right.
\end{aligned}
$$

Throughout this paper, we define $e_{n}=\{0,0, \ldots, 1,0,0, \ldots\}$ where 1 appears at the $n$th place for all $n \in \mathbb{N}$, the sequence $\left(q_{i}\right)$ is a bounded sequence of positive numbers, and the following well-known inequality [5]: $\left|a_{i}+b_{i}\right|^{q_{i}} \leq H\left(\left|a_{i}\right|^{q_{i}}+\left|b_{i}\right|^{q_{i}}\right)$, where $H=2^{h-1}, h=$ $\sup _{i} q_{i}$, and $q_{i} \geq 1$ for every $i \in \mathbb{N}$, is used.

\section{Main results}

In this part, we give the concept of pre-quasi norm on Orlicz sequence space, which is more general than the usual norm, and give the conditions for Orlicz sequence space equipped with the pre-quasi norm to be a Banach space.

Definition 3.1 Let $\mathbb{E}$ be special space of sequences (sss). If there is a function $\varrho: \mathbb{E} \rightarrow$ $[0, \infty[$ fulfilling the following conditions:

(i) $\varrho(x) \geq 0$ for each $x \in \mathbb{E}$ and $\varrho(x)=0 \Leftrightarrow x=\theta$, where $\theta$ is the zero element of $\mathbb{E}$;

(ii) There exists $L \geq 1$ such that $\varrho(\lambda x) \leq L|\lambda| \varrho(x)$ for all $x \in \mathbb{E}$ and for any scalar $\lambda$;

(iii) For some $K \geq 1$, we have $\varrho(x+y) \leq K(\varrho(x)+\varrho(y))$ for every $x, y \in \mathbb{E}$.

The space $\mathbb{E}$ with $\varrho$ is called pre-quasi normed (sss) and is denoted by $\mathbb{E}_{\varrho}$, which gives a class more general than the quasi normed space. If the space $\mathbb{E}$ is complete with $\varrho$, then $\mathbb{E}_{\varrho}$ is called a pre-quasi Banach (sss).

\section{Theorem 3.2 Every quasi-norm is pre-quasi norm.}

Theorem 3.3 If $M$ is an Orlicz function satisfying $\Delta_{2}$-condition, then $\left(\ell_{M}\right)_{\varrho}$ is a pre-quasi Banach (sss), where $\varrho(x)=\sum_{k=0}^{\infty} M\left(\left|x_{k}\right|\right)$ for all $x \in \ell_{M}$.

Proof The function $\varrho: l_{M} \rightarrow\left[0, \infty\left[\right.\right.$, where $\varrho(x)=\sum_{k=0}^{\infty} M\left(\left|x_{k}\right|\right)$ for all $x \in \ell_{M}$, satisfies the following conditions:

(i) $\varrho(x) \geq 0$ for each $x \in \ell_{M}$ and $\varrho(x)=0 \Leftrightarrow x=\theta$, where $\theta$ is the zero element of $\ell_{M}$.

(ii) Assume $\lambda \in \mathbb{R}, x \in l_{M}$, and since $M$ satisfies $\Delta_{2}$-condition, we get a number $a>0$ such that

$$
\varrho(\lambda x)=\sum_{n=0}^{\infty} M\left(\left|\lambda x_{n}\right|\right) \leq|\lambda| a \sum_{n=0}^{\infty} M\left(\left|x_{n}\right|\right)=L|\lambda| \varrho(x),
$$

where $L=\max \{1, a\}$.

(iii) Let $x, y \in l_{M}$. Since $M$ is nondecreasing, convex, and satisfying $\Delta_{2}$-condition, then there exists a number $a>0$ such that

$$
\begin{aligned}
\varrho(x+y) & =\sum_{n=0}^{\infty} M\left(\left|x_{n}+y_{n}\right|\right) \leq \sum_{n=0}^{\infty} M\left(\left|x_{n}\right|+\left|y_{n}\right|\right) \leq \frac{1}{2} \sum_{n=0}^{\infty}\left[M\left(2\left|x_{n}\right|\right)+M\left(2\left|y_{n}\right|\right)\right] \\
& \leq \frac{a}{2}\left(\sum_{n=0}^{\infty} M\left(\left|x_{n}\right|\right)+\sum_{n=0}^{\infty} M\left(\left|y_{n}\right|\right)\right)=K(\varrho(x)+\varrho(y))
\end{aligned}
$$


for some $K=\max \left\{1, \frac{a}{2}\right\}$. Hence $\left(\ell_{M}\right)_{\varrho}$ is a pre-quasi normed (sss). Since $M$ is continuous and nondecreasing, hence $M^{-1}$ exists. To prove that $\left(\ell_{M}\right)_{\varrho}$ is a pre-quasi Banach (sss), suppose $x^{n}=\left(x_{k}^{n}\right)_{k=0}^{\infty}$ to be a Cauchy sequence in $\left(\ell_{M}\right)_{\varrho}$, then for every $\varepsilon>0$, there exists a natural number $n_{0} \in \mathbb{N}$ such that, for all $n, m \geq n_{0}$, one has

$$
\begin{aligned}
& \varrho\left(x^{n}-x^{m}\right)=\sum_{k=0}^{\infty} M\left(\left|x_{k}^{n}-x_{k}^{m}\right|\right)<\varepsilon \\
& \quad \Rightarrow \quad M\left(\left|x_{k}^{n}-x_{k}^{m}\right|\right)<\varepsilon \Rightarrow\left|x_{k}^{n}-x_{k}^{m}\right|<M^{-1}(\varepsilon) .
\end{aligned}
$$

So $\left(x_{k}^{m}\right)$ is a Cauchy sequence in $\mathbb{R}$ for fixed $k \in \mathbb{N}$, this gives $\lim _{m \rightarrow \infty} x_{k}^{m}=x_{k}^{0}$ for fixed $k \in \mathbb{N}$. Hence $\varrho\left(x^{n}-x^{0}\right)<\varepsilon$. Finally, to prove that $x^{0} \in \ell_{M}$, we have

$$
\begin{aligned}
\varrho\left(x^{0}\right) & =\sum_{k=0}^{\infty} M\left(\left|x_{k}^{0}-x_{k}^{n}+x_{k}^{n}\right|\right) \leq K\left(\sum_{k=0}^{\infty} M\left(\left|x_{k}^{0}-x_{k}^{n}\right|\right)+\sum_{k=0}^{\infty} M\left(\left|x_{k}^{n}\right|\right)\right) \\
& =K\left(\varrho\left(x^{n}-x^{0}\right)+\varrho\left(x^{n}\right)\right)<\infty,
\end{aligned}
$$

so $x^{0} \in \ell_{M}$. This means that $\left(\ell_{M}\right)_{\varrho}$ is a pre-quasi Banach (sss).

Corollary 3.4 $\left(\ell^{p}\right)_{\varrho}$, where $\varrho(x)=\sum_{i=0}^{\infty}\left|x_{i}\right|^{p}$ for all $x \in \ell^{p}$ is a pre-quasi Banach (sss), if $0<p<\infty$.

\section{Multiplication operator on pre-quasi normed (sss)}

In this part, we define a multiplication operator on Orlicz sequence space with a pre-quasi norm and give the necessity and sufficient conditions on Orlicz sequence space equipped with the pre-quasi norm such that the multiplication operator defined on Orlicz sequence space is a bounded, approximable, invertible, Fredholm, and closed range operator.

Definition 4.1 Let $\alpha: \mathbb{N} \rightarrow \mathbb{C}$ be a bounded sequence and $E_{\varrho}$ be a pre-quasi normed (sss), the multiplication operator is defined as $T_{\alpha}: E \rightarrow E$, where $T_{\alpha} x=\alpha x=\left(\alpha_{k} x_{k}\right)_{k=0}^{\infty}$ for all $x \in E$. If $T_{\alpha}$ is continuous, we call it a multiplication operator induced by $\alpha$.

Theorem 4.2 If $\alpha: \mathbb{N} \rightarrow \mathbb{C}$ is a mapping and $M$ is an Orlicz function satisfying $\Delta_{2}$ condition, then $\alpha \in \ell_{\infty}$ if and only if $T_{\alpha} \in L\left(\left(\ell_{M}\right)_{\varrho}\right)$, where $\varrho(x)=\sum_{k=0}^{\infty} M\left(\left|x_{k}\right|\right)$ for all $x \in \ell_{M}$.

Proof Let $\alpha \in \ell_{\infty}$. Then there exists $C>0$ such that $\left|\alpha_{n}\right| \leq C$ for all $n \in \mathbb{N}$. For $x \in\left(\ell_{M}\right)_{\varrho}$, since $M$ is nondecreasing and satisfying $\Delta_{2}$-condition, we have

$$
\begin{aligned}
\varrho\left(T_{\alpha} x\right) & =\varrho(\alpha x)=\varrho\left(\left(\alpha_{k} x_{k}\right)_{k=0}^{\infty}\right)=\sum_{k=0}^{\infty} M\left(\left|\alpha_{k}\right|\left|x_{k}\right|\right) \leq \sum_{k=0}^{\infty} M\left(C\left|x_{k}\right|\right) \\
& \leq D \sum_{k=0}^{\infty} M\left(\left|x_{k}\right|\right)=D \varrho(x),
\end{aligned}
$$

where $D$ is a constant depending on $C$, which implies that $T_{\alpha} \in L\left(\left(\ell_{M}\right)_{\varrho}\right)$. 
Conversely, suppose that $T_{\alpha} \in L\left(\left(\ell_{M}\right)_{\varrho}\right)$. We prove that $\alpha \in \ell_{\infty}$. For, if $\alpha$ is not a bounded function, then for every $n \in \mathbb{N}$, there exists some $i_{n} \in \mathbb{N}$ such that $\alpha_{i_{n}}>n$. Since $M$ is nondecreasing, we obtain

$$
\begin{aligned}
\varrho\left(T_{\alpha} e_{i_{n}}\right)=\varrho\left(\alpha e_{i_{n}}\right) & =\varrho\left(\left(\alpha_{k}\left(e_{i_{n}}\right)_{k}\right)_{k=0}^{\infty}\right)=\sum_{k=0}^{\infty} M\left(\left|\alpha_{k}\right|\left|\left(e_{i_{n}}\right)_{k}\right|\right) \\
& =M\left(\left|\alpha_{i_{n}}\right|\right)>M(n)=M(n) \varrho\left(e_{i_{n}}\right) .
\end{aligned}
$$

This proves that $T_{\alpha}$ is not a bounded operator. Hence, $\alpha$ must be a bounded function.

Theorem 4.3 Let $\alpha: \mathbb{N} \rightarrow \mathbb{C}$ be a mapping and $\left(\ell_{M}\right)_{\varrho}$ be a pre-quasi normed (sss), where $\varrho(x)=\sum_{k=0}^{\infty} M\left(\left|x_{k}\right|\right)$ for all $x \in \ell_{M} .\left|\alpha_{n}\right|=1$ for all $n \in \mathbb{N}$ if and only if $T_{\alpha}$ is an isometry.

Proof Let $\left|\alpha_{n}\right|=1$ for all $n \in \mathbb{N}$. Then

$$
\varrho\left(T_{\alpha} x\right)=\varrho(\alpha x)=\varrho\left(\left(\alpha_{k} x_{k}\right)_{k=0}^{\infty}\right)=\sum_{k=0}^{\infty} M\left(\left|\alpha_{k}\right|\left|x_{k}\right|\right)=\sum_{k=0}^{\infty} M\left(\left|x_{k}\right|\right)=\varrho(x)
$$

for all $x \in\left(\ell_{M}\right)_{\varrho}$. Hence $T_{\alpha}$ is an isometry.

Conversely, suppose that $\left|\alpha_{n}\right|<1$ for some $n=n_{0}$. Since $M$ is nondecreasing, we have

$$
\varrho\left(T_{\alpha} e_{n_{0}}\right)=\varrho\left(\alpha e_{n_{0}}\right)=\varrho\left(\left(\alpha_{k}\left(e_{n_{0}}\right)_{k}\right)_{k=0}^{\infty}\right)=\sum_{k=0}^{\infty} M\left(\left|\alpha_{k}\right|\left|\left(e_{n_{0}}\right)_{k}\right|\right)=M\left(\alpha_{n_{0}}\right)<\varrho\left(e_{n_{0}}\right) .
$$

Similarly, if $\left|\alpha_{n_{0}}\right|>1$, then we can show that $\varrho\left(T_{\alpha} e_{n_{0}}\right)>\varrho\left(e_{n_{0}}\right)$. In both cases, we get contradiction. Hence, $\left|\alpha_{n}\right|=1$ for all $n \in \mathbb{N}$.

Theorem 4.4 Let $\alpha: \mathbb{N} \rightarrow \mathbb{C}$ be a mapping, $M$ be an Orlicz function satisfying $\Delta_{2}$ condition, and $T_{\alpha} \in L\left(\left(\ell_{M}\right)_{\varrho}\right)$, where $\varrho(x)=\sum_{k=0}^{\infty} M\left(\left|x_{k}\right|\right)$ for all $x \in \ell_{M}$. Then $T_{\alpha} \in$ $\Psi\left(\left(\ell_{M}\right)_{\varrho}\right)$ if and only if $\left(\alpha_{n}\right)_{n=0}^{\infty} \in C_{0}$.

Proof Suppose that $T_{\alpha}$ is an approximable operator, hence $T_{\alpha}$ is a compact operator. We show that $\lim _{n \rightarrow \infty} \alpha_{n}=0$. For if this were not true, then there exists $\delta>0$ such that the set $B_{\delta}=\left\{r \in \mathbb{N}:\left|\alpha_{r}\right| \geq \delta\right\}$ is an infinite set. Let $d_{1}, d_{2}, \ldots, d_{n}, \ldots$ be in $B_{\delta}$. Then $\left\{e_{d_{n}}: d_{n} \in B_{\delta}\right\}$ is an infinite bounded set in $\left(\ell_{M}\right)_{\varrho}$. Consider

$$
\begin{aligned}
\varrho\left(T_{\alpha} e_{d_{n}}-T_{\alpha} e_{d_{m}}\right) & \left.=\varrho\left(\alpha e_{d_{n}}-\alpha e_{d_{m}}\right)=\varrho\left(\left(\alpha_{k}\left(\left(e_{d_{n}}\right)_{k}-\left(e_{p_{m}}\right)\right)_{k}\right)\right)_{k=0}^{\infty}\right) \\
& =\sum_{k=0}^{\infty} M\left(\left|\alpha_{k}\left(\left(e_{d_{n}}\right)_{k}-\left(e_{p_{m}}\right)_{k}\right)\right|\right) \\
& \geq \sum_{k=0}^{\infty} M\left(\delta\left|\left(\left(e_{d_{n}}\right)_{k}-\left(e_{p_{m}}\right)_{k}\right)\right|\right)=\varrho\left(\delta e_{d_{n}}-\delta e_{d_{m}}\right)
\end{aligned}
$$

for all $d_{n}, d_{m} \in B_{\delta}$. This proves that $\left\{e_{d_{n}}: d_{n} \in B_{\delta}\right\}$ is a bounded sequence which cannot have a convergent subsequence under $T_{\alpha}$. This shows that $T_{\alpha}$ cannot be compact, hence 
it is not an approximable operator, which is a contradiction. Hence, $\lim _{n \rightarrow \infty} \alpha_{n}=0$. Conversely, suppose $\lim _{n \rightarrow \infty} \alpha_{n}=0$. Then, for every $\delta>0$, the set $B_{\delta}=\left\{n \in \mathbb{N}:\left|\alpha_{n}\right| \geq \delta\right\}$ is a finite set. Then

$$
\left(\left(\ell_{M}\right)_{\varrho}\right)_{B_{\delta}}=\left\{x=\left(x_{n}\right) \in \omega: n \in B_{\delta}\right\}
$$

is a finite dimensional space for each $\delta>0$. Therefore, $T_{\alpha} \mid\left(\left(\ell_{M}\right)_{\varrho}\right)_{B_{\delta}}$ is a finite rank operator. For each $n \in \mathbb{N}$, define $\alpha_{n}: \mathbb{N} \rightarrow \mathbb{C}$ by

$$
\left(\alpha_{n}\right)_{m}= \begin{cases}\alpha_{m} & m \in B_{\frac{1}{n}} \\ 0 & \text { otherwise }\end{cases}
$$

Clearly, $T_{\alpha_{n}}$ is a finite rank operator as the space $\left(\left(\ell_{M}\right)_{\varrho}\right)_{B_{\frac{1}{n}}}$ is finite dimensional for each $n \in \mathbb{N}$. Now, since $M$ is convex and nondecreasing, we have

$$
\begin{aligned}
\varrho\left(\left(T_{\alpha}-T_{\alpha_{n}}\right) x\right) & =\varrho\left(\left(\left(\alpha_{m}-\left(\alpha_{n}\right)_{m}\right) x_{m}\right)_{m=0}^{\infty}\right) \\
& =\sum_{m=0}^{\infty} M\left(\left|\left(\alpha_{m}-\left(\alpha_{n}\right)_{m}\right) x_{m}\right|\right) \\
& =\sum_{m=0, m \in B_{\frac{1}{n}}}^{\infty} M\left(\left|\left(\alpha_{m}-\left(\alpha_{n}\right)_{m}\right) x_{m}\right|\right)+\sum_{m=0, m \notin B_{\frac{1}{n}}}^{\infty} M\left(\left|\left(\alpha_{m}-\left(\alpha_{n}\right)_{m}\right) x_{m}\right|\right) \\
& =\sum_{m=0, m \notin B_{\frac{1}{n}}}^{\infty} M\left(\left|\alpha_{m} x_{m}\right|\right) \leq \frac{1}{n} \sum_{m=0, m \notin B}^{\infty} M\left(\left|x_{m}\right|\right)<\frac{1}{n} \sum_{m=0}^{\infty} M\left(\left|x_{m}\right|\right) \\
& =\frac{1}{n} \varrho(x) .
\end{aligned}
$$

This proves that $\left\|T_{\alpha}-T_{\alpha_{n}}\right\| \leq \frac{1}{n}$ and that $T_{\alpha}$ is a limit of finite rank operators and hence, $T_{\alpha}$ is an approximable operator.

Theorem 4.5 Let $\alpha: \mathbb{N} \rightarrow \mathbb{C}$ be a mapping, $M$ be an Orlicz function satisfying $\Delta_{2}$ condition, and $T_{\alpha} \in L\left(\left(\ell_{M}\right)_{\varrho}\right)$, where $\varrho(x)=\sum_{k=0}^{\infty} M\left(\left|x_{k}\right|\right)$ for all $x \in \ell_{M}$. Then $T_{\alpha} \in$ $L_{c}\left(\left(\ell_{M}\right)_{\varrho}\right)$ if and only if $\left(\alpha_{n}\right)_{n=0}^{\infty} \in C_{0}$.

Proof It is easy, so omitted.

Corollary 4.6 Let $M$ be an Orlicz function satisfying $\Delta_{2}$-condition, we have

$$
L_{c}\left(\left(\ell_{M}\right)_{\varrho}\right) \varsubsetneqq L\left(\left(\ell_{M}\right)_{\varrho}\right),
$$

where $\varrho(x)=\sum_{k=0}^{\infty} M\left(\left|x_{k}\right|\right)$ for all $x \in \ell_{M}$.

Proof Since the identity operator $I$ on $\left(\ell_{M}\right)_{\varrho}$ is a multiplication operator induced by the sequence $\alpha=(1,1, \ldots)$, hence $I \notin L_{c}\left(\left(\ell_{M}\right)_{\varrho}\right)$ and $I \in L\left(\left(\ell_{M}\right)_{\varrho}\right)$. 
Theorem 4.7 Let $\left(\ell_{M}\right)_{\varrho}$ be a pre-quasi Banach (sss), where $\varrho(x)=\sum_{k=0}^{\infty} M\left(\left|x_{k}\right|\right)$ for all $x \in \ell_{M}$ and $T_{\alpha} \in L\left(\left(\ell_{M}\right)_{\varrho}\right)$. Then $\alpha$ is bounded away from zero on $\mathbb{N} \backslash \operatorname{ker}(\alpha):=\operatorname{ker}(\alpha)^{c}$ if and only if $T_{\alpha}$ has closed range.

Proof Suppose that $\alpha$ is bounded away from zero on $\operatorname{ker}(\alpha)^{c}$. Then there exists $\epsilon>0$ such that $\left|\alpha_{n}\right| \geq \epsilon$ for all $n \in \operatorname{ker}(\alpha)^{c}$. We have to prove that range $\left(T_{\alpha}\right)$ is closed. Let $z$ be a limit point of range $\left(T_{\alpha}\right)$. Then there exists a sequence $T_{\alpha} x_{n}$ in $\left(\ell_{M}\right)_{\varrho}$, for all $n \in \mathbb{N}$ such that $\lim _{n \rightarrow \infty} T_{\alpha} x_{n}=z$. Clearly, the sequence $T_{\alpha} x_{n}$ is a Cauchy sequence. Now, since $M$ is nondecreasing, we have

$$
\begin{aligned}
\varrho\left(T_{\alpha} x_{n}-T_{\alpha} x_{m}\right)= & \sum_{k=0}^{\infty} M\left(\left|\alpha_{k}\left(x_{n}\right)_{k}-\alpha_{k}\left(x_{m}\right)_{k}\right|\right) \\
= & \sum_{k=0, k \in \operatorname{ker}(\alpha)^{c}}^{\infty} M\left(\left|\alpha_{k}\left(x_{n}\right)_{k}-\alpha_{k}\left(x_{m}\right)_{k}\right|\right) \\
& +\sum_{k=0, k \notin \operatorname{ker}(\alpha)^{c}}^{\infty} M\left(\left|\alpha_{k}\left(x_{n}\right)_{k}-\alpha_{k}\left(x_{m}\right)_{k}\right|\right) \\
\geq & \sum_{k=0, k \in \operatorname{ker}(\alpha)^{c}}^{\infty} M\left(\left|\alpha_{k}\right|\left|\left(x_{n}\right)_{k}-\left(x_{m}\right)_{k}\right|\right) \\
= & \sum_{k=0}^{\infty} M\left(\left|\alpha_{k}\right|\left|\left(y_{n}\right)_{k}-\left(y_{m}\right)_{k}\right|\right) \\
> & \sum_{k=0}^{\infty} M\left(\left|\epsilon\left(y_{n}\right)_{k}-\epsilon\left(y_{m}\right)_{k}\right|\right)=\varrho\left(\epsilon\left(y_{n}-y_{m}\right)\right),
\end{aligned}
$$

where

$$
\left(y_{n}\right)_{k}= \begin{cases}\left(x_{n}\right)_{k}, & k \in \operatorname{ker}(\alpha)^{c} \\ 0, & k \notin \operatorname{ker}(\alpha)^{c}\end{cases}
$$

This proves that $\left\{y_{n}\right\}$ is a Cauchy sequence in $\left(\ell_{M}\right)_{\varrho}$. But $\left(\ell_{M}\right)_{\varrho}$ is complete. Therefore, there exists $x \in\left(\ell_{M}\right)_{\varrho}$ such that $\lim _{n \rightarrow \infty} y_{n}=x$. In view of the continuity of $T_{\alpha}, \lim _{n \rightarrow \infty} T_{\alpha} y_{n}=$ $T_{\alpha} x$. But $\lim _{n \rightarrow \infty} T_{\alpha} x_{n}=\lim _{n \rightarrow \infty} T_{\alpha} y_{n}=z$. Therefore, $T_{\alpha} x=z$. Hence $z \in \operatorname{range}\left(T_{\alpha}\right)$. This proves that $T_{\alpha}$ has closed range. Conversely, suppose that $T_{\alpha}$ has closed range. Then $T_{\alpha}$

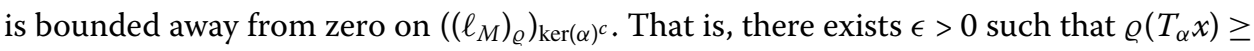
$\varrho(\epsilon x)$ for all $x \in\left(\left(\ell_{M}\right)_{\varrho}\right)_{\operatorname{ker}(\alpha)^{c}}$. Let $D=\left\{k \in \operatorname{ker}(\alpha)^{c}:\left|\alpha_{k}\right|<\epsilon\right\}$. If $D \neq \phi$, then for $n_{0} \in D$, we have

$$
\left.\left.\left.\left.\varrho\left(T_{\alpha} e_{n_{0}}\right)=\varrho\left(\left(\alpha_{k}\left(e_{n_{0}}\right)\right)_{k}\right)\right)_{k=0}^{\infty}\right)=\sum_{k=0}^{\infty} M\left(\mid \alpha_{k}\left(e_{n_{0}}\right)_{k}\right) \mid\right)<\sum_{k=0}^{\infty} M\left(\mid \epsilon\left(e_{n_{0}}\right)_{k}\right) \mid\right)=\varrho\left(\epsilon e_{n_{0}}\right),
$$

which is a contradiction. Hence, $D=\phi$ so that $\left|\alpha_{k}\right| \geq \epsilon$ for all $k \in \operatorname{ker}(\alpha)^{c}$. This proves the theorem. 
Theorem 4.8 Let $\alpha: \mathbb{N} \rightarrow \mathbb{C}$ be a mapping and $\left(\ell_{M}\right)_{e}$ be a pre-quasi Banach (sss), where $\varrho(x)=\sum_{k=0}^{\infty} M\left(\left|x_{k}\right|\right)$ for all $x \in \ell_{M}$. There exist $a>0$ and $A>0$ such that $a<\alpha_{n}<A$; for all $n \in \mathbb{N}$ if and only if $T_{\alpha} \in L\left(\left(\ell_{M}\right)_{\varrho}\right)$ is invertible.

Proof Suppose that the condition is true. Define $\beta: \mathbb{N} \rightarrow \mathbb{C}$ by $\beta_{n}=\frac{1}{\alpha_{n}}$. Then $T_{\alpha}$ and $T_{\beta}$ are bounded linear operators in view of Theorem 4.2. Also $T_{\alpha} \cdot T_{\beta}=T_{\beta} \cdot T_{\alpha}=I$. Hence, $T_{\beta}$ is the inverse of $T_{\alpha}$. Conversely, suppose that $T_{\alpha}$ is invertible. Then range $\left(T_{\alpha}\right)=\left(\left(\ell_{M}\right)_{\varrho}\right)_{\mathbb{N}}$. Therefore, range $\left(T_{\alpha}\right)$ is closed. Hence, by Theorem 4.7, there exists $a>0$ such that $\left|\alpha_{n}\right| \geq a$ for all $n \in \operatorname{ker}(\alpha)^{c}$. Now $\operatorname{ker}(\alpha)=\phi$; otherwise $\alpha_{n_{0}}=0$ for some $n_{0} \in \mathbb{N}$, in which case $e_{n_{0}} \in$ $\operatorname{ker}\left(T_{\alpha}\right)$, which is a contradiction, since $\operatorname{ker}\left(T_{\alpha}\right)$ is trivial. Hence, $\left|\alpha_{n}\right| \geq a$ for all $n \in \mathbb{N}$. Since $T_{\alpha}$ is bounded, so by Theorem 4.2, there exists $A>0$ such that $\left|\alpha_{n}\right| \leq A$ for all $n \in \mathbb{N}$. Thus, we have proved that $a \leq\left|\alpha_{n}\right| \leq A$ for all $n \in \mathbb{N}$.

Theorem 4.9 Let $\left(\ell_{M}\right)_{\varrho}$ be a pre-quasi Banach (sss), where $\varrho(x)=\sum_{k=0}^{\infty} M\left(\left|x_{k}\right|\right)$ for all $x \in \ell_{M}$ and $T_{\alpha} \in L\left(\left(\ell_{M}\right)_{\varrho}\right)$. Then $T_{\alpha}$ is a Fredholm operator if and only if

(i) $\operatorname{ker}(\alpha)$ is a finite subset of $\mathbb{N}$.

(ii) $\left|\alpha_{n}\right| \geq \epsilon$ for all $n \in \operatorname{ker}(\alpha)^{c}$.

Proof Suppose that $T_{\alpha}$ is Fredholm. If $\operatorname{ker}(\alpha)$ is an infinite subset of $\mathbb{N}$, then $e_{n} \in \operatorname{ker}\left(T_{\alpha}\right)$ for all $n \in \operatorname{ker}(\alpha)$. But $e_{n}$ s are linearly independent, which shows that $\operatorname{ker}\left(T_{\alpha}\right)$ is infinite dimensional, which is a contradiction. Hence, $\operatorname{ker}(\alpha)$ must be a finite subset of $\mathbb{N}$. Condition (ii) follows from Theorem 4.7. Conversely, if conditions (i) and (ii) are true, then we prove that $T_{\alpha}$ is Fredholm. In view of Theorem 4.7, condition (ii) implies that $T_{\alpha}$ has closed range. Condition (i) implies that $\operatorname{ker}\left(T_{\alpha}\right)$ and $\left(\operatorname{range}\left(T_{\alpha}\right)\right)^{c}$ are finite dimensional. This proves that $T_{\alpha}$ is Fredholm.

\section{Pre-quasi closed ideal components}

For which Orlicz sequence space $\ell_{M}$, are the components of pre-quasi operator ideal $S_{\ell_{M}}$ closed?

Theorem $5.1\left(S_{\left(\ell_{M}\right)_{\rho}}, g\right)$ is a pre-quasi closed operator ideal, where $\rho(x)=\sum_{n=0}^{\infty} M\left(\left|x_{n}\right|\right)$ and $g(T)=\rho\left(\left(s_{n}(T)\right)_{n=0}^{\infty}\right)$, if $X, Y$ are normed spaces and $M$ is an Orlicz function satisfying $\Delta_{2}$-condition.

Proof Since $\ell_{M}$ is a pre-modular (sss) by Theorem 2.13, then from Theorem 2.16, the function $g(T)=\rho\left(\left(s_{n}(T)\right)_{n=0}^{\infty}\right)$ is a pre-quasi norm on $S_{\left(\ell_{M}\right)_{\rho}}$. Let $T_{m} \in S_{\left(\ell_{M}\right)_{\rho}}(X, Y)$ for all $m \in \mathbb{N}$ and $\lim _{m \rightarrow \infty} g\left(T_{m}-T\right)=0$, then by using Definition 2.12(vii) there exists a constant $\xi>0$, and since $L(X, Y) \supseteq S_{\left(\ell_{M}\right)_{\rho}}(X, Y)$, we get

$$
g\left(T-T_{m}\right)=\sum_{n=0}^{\infty} M\left(s_{n}\left(T-T_{m}\right)\right) \geq \xi\left\|T-T_{m}\right\| M(1),
$$

then $\left(T_{m}\right)_{m \in \mathbb{N}}$ is a convergent sequence in $L(X, Y)$. While $\left(s_{n}\left(T_{m}\right)\right)_{n=0}^{\infty} \in\left(\ell_{M}\right)_{\rho}$ for each $m \in$ $\mathbb{N}$, hence using Definition 2.12 conditions (iii), (iv), and $M$ is continuous from right at 0 , we obtain

$$
g(T)=\sum_{n=0}^{\infty} M\left(s_{n}(T)\right)=\sum_{n=0}^{\infty} M\left(s_{n}\left(T-T_{m}+T_{m}\right)\right)
$$




$$
\begin{aligned}
& \leq K\left(\sum_{n=0}^{\infty} M\left(s_{\left[\frac{n}{2}\right]}\left(T-T_{m}\right)\right)+\sum_{n=0}^{\infty} M\left(s_{\left[\frac{n}{2}\right]}\left(T_{m}\right)\right)\right) \\
& \leq K\left(\sum_{n=0}^{\infty} M\left(\left\|T_{m}-T\right\|\right)+K_{0} \sum_{n=0}^{\infty} M\left(s_{n}\left(T_{m}\right)\right)\right)<\varepsilon,
\end{aligned}
$$

we have $\left(s_{n}(T)\right)_{n=0}^{\infty} \in\left(\ell_{M}\right)_{\rho}$, then $T \in S_{\left(\ell_{M}\right)_{\rho}}(X, Y)$.

\section{Pre-quasi simple Banach operator ideal}

We give here the sufficient conditions on Orlicz sequence space such that the pre-quasi operator ideal formed by the sequence of $s$-numbers and this sequence space are strictly contained for different Orlicz functions.

Theorem 6.1 Let $\varphi_{1}$ and $\varphi_{2}$ be two Orlicz functions satisfying $\Delta_{2}$-condition. For any infinite dimensional Banach spaces $X, Y$ and $\varphi_{2}(t)<\varphi_{1}(t)$ for all $t \in(0, \infty)$, then

$$
S_{\ell_{\varphi_{1}}}(X, Y) \varsubsetneqq S_{\ell_{\varphi_{2}}}(X, Y) \varsubsetneqq L(X, Y) .
$$

Proof Let $X$ and $Y$ be infinite dimensional Banach spaces, if $T \in S_{\ell_{\varphi_{1}}}(X, Y)$, then $\left(s_{n}(T)\right) \in$ $\ell_{\varphi_{1}}$. We have

$$
\sum_{n=0}^{\infty} \varphi_{2}\left(s_{n}(T)\right)<\sum_{n=0}^{\infty} \varphi_{1}\left(s_{n}(T)\right)<\infty
$$

hence $T \in S_{\ell_{\varphi_{2}}}(X, Y)$. Next, if we take $\left(s_{n}(T)\right)_{n=0}^{\infty}$ such that $\varphi_{1}\left(s_{n}(T)\right)=\frac{1}{\sqrt[p]{n+1}}$ and $\varphi_{2}\left(s_{n}(T)\right)=$ $\frac{1}{(n+1) \frac{q}{p}}$ for any $q>p>0$, we can find $T \in L(X, Y)$ with $T$ does not belong to $S_{\ell_{\varphi_{1}}}(X, Y)$ and $T \in S_{\ell_{\varphi_{2}}}(X, Y)$.

It is easy to verify that $S_{\ell_{\varphi_{2}}}(X, Y) \subset L(X, Y)$. Next, if we take $\left(s_{n}(T)\right)_{n=0}^{\infty}$ such that $\varphi_{2}\left(s_{n}(T)\right)=\frac{1}{n+1}$, one can find $T \in L(X, Y)$ such that $T$ does not belong to $S_{\ell_{\varphi_{2}}}(X, Y)$. This completes the proof.

Corollary 6.2 For any infinite dimensional Banach spaces $X, Y$ and $0<p<q<\infty$, then

$$
S_{\ell_{p}}(X, Y) \varsubsetneqq S_{\ell_{q}}(X, Y) \varsubsetneqq L(X, Y) .
$$

The following question arises naturally: For which Orlicz sequence space, is the prequasi Banach ideal simple?

Theorem 6.3 Let $\varphi_{1}$ and $\varphi_{2}$ be two Orlicz functions satisfying $\Delta_{2}$-condition. For any infinite dimensional Banach spaces $X, Y$ and $\varphi_{2}(t)<\varphi_{1}(t)$ for all $t \in(0, \infty)$, then

$$
L\left(S_{\ell_{\varphi_{2}}}, S_{\ell_{\varphi_{1}}}\right)=\Psi\left(S_{\ell_{\varphi_{2}}}, S_{\ell_{\varphi_{1}}}\right) .
$$

Proof Suppose that there exists $T \in L\left(S_{\ell_{\varphi_{2}}}, S_{\ell_{\varphi_{1}}}\right)$ which is not approximable. According to Lemma 2.3, we can find $X \in L\left(S_{\ell_{\varphi_{2}}}, S_{\ell_{\varphi_{2}}}\right)$ and $B \in L\left(S_{\ell_{\varphi_{1}}}, S_{\ell_{\varphi_{1}}}\right)$ with $B T X I_{k}=I_{k}$. Then it follows for all $k \in \mathbb{N}$ that

$$
\left\|I_{k}\right\|_{S_{\ell_{1}}}=\sum_{n=0}^{\infty} \varphi_{1}\left(s_{n}\left(I_{k}\right)\right) \leq\|B T X\|\left\|I_{k}\right\|_{S_{\varphi_{2}}} \leq \sum_{n=0}^{\infty} \varphi_{2}\left(s_{n}\left(I_{k}\right)\right) .
$$


This contradicts Theorem 6.1, which is a contradiction. Hence $T \in \Psi\left(S_{\ell_{\varphi_{2}}}, S_{\ell_{\varphi_{1}}}\right)$, which finishes the proof.

Corollary 6.4 Let $\varphi_{1}$ and $\varphi_{2}$ be two Orlicz functions satisfying $\Delta_{2}$-condition. For any infinite dimensional Banach spaces $X, Y$ and $\varphi_{2}(t)<\varphi_{1}(t)$ for all $t \in(0, \infty)$, then

$$
L\left(S_{\ell_{\varphi_{2}}}, S_{\ell_{\varphi_{1}}}\right)=L_{C}\left(S_{\ell_{\varphi_{2}}}, S_{\ell_{\varphi_{1}}}\right) .
$$

Proof Since every approximable operator is compact.

\section{Eigenvalues of s-type operators}

We give here the sufficient conditions on Orlicz sequence space such that the pre-quasi operator ideal is formed by the sequence of $s$-numbers and this sequence space is strictly contained in the class of all bounded linear operators whose sequence of eigenvalues belongs to this sequence space.

\section{Notations 7.1}

$$
\begin{aligned}
& S_{E}^{\lambda}:=\left\{S_{E}^{\lambda}(X, Y) ; X \text { and } Y \text { are Banach spaces }\right\}, \text { where } \\
& S_{E}^{\lambda}(X, Y):=\left\{T \in L(X, Y):\left(\left(\lambda_{i}(T)\right)_{n=0}^{\infty} \in E \text { and }\left\|T-\lambda_{n}(T)\right\|\right.\right.
\end{aligned}
$$

is not invertible for all $n \in \mathbb{N}\}$.

Theorem 7.2 For any infinite dimensional Banach spaces $X$ and $Y$. If $M$ is an Orlicz function satisfying $\Delta_{2}$-condition, then

$$
S_{\ell_{M}}(X, Y) \varsubsetneqq S_{\ell_{M}}^{\lambda}(X, Y) .
$$

Proof Let $T \in S_{\ell_{M}}(X, Y)$, then $\left(s_{n}(T)\right)_{n=0}^{\infty} \in \ell_{M}$, we have $\sum_{n=0}^{\infty} M\left(s_{n}(T)\right)<\infty$, and since $M$ is continuous, so $\lim _{n \rightarrow \infty} s_{n}(T)=0$. Suppose $\left\|T-s_{n}(T)\right\|$ is invertible for all $n \in \mathbb{N}$, then $\left\|T-s_{n}(T)\right\|^{-1}$ exists and is bounded for all $n \in \mathbb{N}$. This gives $\lim _{n \rightarrow \infty}\left\|T-s_{n}(T)\right\|^{-1}=\|T\|^{-1}$ exists and is bounded. Since $\left(S_{\ell_{M}}, g\right)$ is a pre-quasi operator ideal, we have

$$
I=T T^{-1} \in S_{\ell_{M}}(X, Y) \quad \Rightarrow \quad\left(s_{n}(I)\right)_{n=0}^{\infty} \in \ell_{M} \quad \Rightarrow \quad \lim _{n \rightarrow \infty} s_{n}(I)=0 .
$$

But $\lim _{n \rightarrow \infty} s_{n}(I)=1$. This is a contradiction, then $\left\|T-s_{n}(T)\right\|$ is not invertible for all $n \in \mathbb{N}$. Therefore the sequence $\left(s_{n}(T)\right)_{n=0}^{\infty}$ is the eigenvalues of $T$. Next, on considering $\left(s_{n}(T)\right)_{n=0}^{\infty}$ such that $M\left(s_{n}(T)\right)=\frac{1}{n+1}$, we find $T \in L(X, Y)$ with $T$ does not belong to $S_{\ell_{M}}(X, Y)$, and if we take $\left(\lambda_{n}(T)\right)_{n=0}^{\infty}$ such that $M\left(\lambda_{n}(T)\right)=\frac{1}{(n+1)^{2}}$, hence $T \in S_{\ell_{M}}^{\lambda}(X, Y)$. This finishes the proof.

Acknowledgements

The authors thank the anonymous referees for their constructive suggestions and helpful comments which led to significant improvement of the original manuscript of this paper.

Funding

Not applicable.

Availability of data and materials Not applicable. 


\section{Competing interests}

The authors declare that they have no competing interests.

\section{Authors' contributions}

All authors contributed equally to the writing of this paper. All authors read and approved the final manuscript.

\section{Publisher's Note}

Springer Nature remains neutral with regard to jurisdictional claims in published maps and institutional affiliations.

Received: 20 November 2019 Accepted: 14 February 2020 Published online: 28 February 2020

\section{References}

1. Abrahmse, M.B.: Multiplication operators. In: Hilbert Space Operators, Lecture Notes in Mathematics, vol. 693, pp. 17-36. Springer, Berlin (1978)

2. Alotaibi, A., Mursaleen, M., Raj, K.: Double sequence spaces by means of Orlicz functions. Abstr. Appl. Anal. 2014, Article ID 260326 (2014)

3. Alotaibi, A., Mursaleen, M., Sharma, S.K.: Double sequence spaces over $n$-normed spaces defined by a sequence of Orlicz functions. J. Inequal. Appl. 2014, 216 (2014)

4. Alotaibi, A., Mursaleen, M., Sharma, S.K., Mohiuddine, S.A.: Sequence spaces of fuzzy numbers defined by a Musielak-Orlicz function. Filomat 29(7), 1461-1468 (2015)

5. Altay, B., Başar, F.: Generalization of the sequence space $\ell(p)$ derived by weighted means. J. Math. Anal. Appl. 330(1), 147-185 (2007)

6. Bakery, A.A., Mohammed, M.M.: Small pre-quasi Banach operator ideals of type Orlicz-Cesàro mean sequence spaces. J. Funct. Spaces 2019, Article ID 7265010 (2019)

7. Et, M., Mursaleen, M., Işik, M.: On a class of fuzzy sets defined by Orlicz functions. Filomat 27(5), 789-796 (2013)

8. Faried, N., Bakery, A.A.: Mappings of type Orlicz and generalized Cesáro sequence space. J. Inequal. Appl. (2013). https://doi.org/10.1186/1029-242X-2013-186

9. Faried, N., Bakery, A.A.: Small operator ideals formed by s numbers on generalized Cesáro and Orlicz sequence spaces. J. Inequal. Appl. (2018). https://doi.org/10.1186/s13660-018-1945-y

10. Komal, B.S., Gupta, S.: Multiplication operators between Orlicz spaces. Integral Equ. Oper. Theory 41(3), 324-330 (2001)

11. Komal, B.S., Pandoh, S., Raj, K.: Multiplication operators on Cesáro sequence spaces. Demonstr. Math. 49(4), 430-436 (2016)

12. Krasnoselskii, M.A., Rutickii, Y.B.: Convex Functions and Orlicz Spaces. Gorningen, Netherlands (1961)

13. Lindenstrauss, J., Tzafriri, L.: On Orlicz sequence spaces. Isr. J. Math. 10, 379-390 (1971)

14. Makarov, B.M., Faried, N.: Some properties of operator ideals constructed by s numbers (in Russian). In: Theory of Operators in Functional Spaces. Academy of Science. Siberian Section, pp. 206-211. Novosibirsk, Russia (1977)

15. Mohiuddine, S.A., Raj, K.: Vector valued Orlicz-Lorentz sequence spaces and their operator ideals. J. Nonlinear Sci. Appl. 10, 338-353 (2017)

16. Mrowka, T:: A Brief Introduction to Linear Analysis: Fredholm Operators. Geometry of Manifolds. Fall 2004 (Massachusetts Institute of Technology: MIT OpenCourseWare) (2004)

17. Mursaleen, M., Noman, A.K.: Compactness by the Hausdorff measure of noncompactness. Nonlinear Anal. 73 2541-2557 (2010)

18. Mursaleen, M., Noman, A.K.: Compactness of matrix operators on some new difference sequence spaces. Linear Algebra Appl. 436, 41-52 (2012)

19. Mursaleen, M., Raj, K., Sharma, S.K.: Some spaces of difference sequences and lacunary statistical convergence in n-normed space defined by sequence of Orlicz functions. Miskolc Math. Notes 16(1), 283-304 (2015)

20. Mursaleen, M., Sharma, S., Kiliçman, A.: Sequence spaces defined by Musielak-Orlicz function over $n$-normed spaces. Abstr. Appl. Anal. 2013, Article ID 364743 (2013)

21. Mursaleen, M., Sharma, S.K., Mohiuddine, S.A., Kiliçman, A.: New difference sequence spaces defined by Musielak-Orlicz function. Abstr. Appl. Anal. 2014, Article ID 691632 (2014)

22. Orlicz, W.: Über Räume ( $\left.L^{M}\right)$. Bull. Int. Acad Polon. Sci. A 93-107 (1936)

23. Pietsch, A.: Small ideals of operators. Stud. Math. 51, 265-267 (1974)

24. Pietsch, A.: Operator Ideals. North-Holland, Amsterdam (1980)

25. Pietsch, A.: Eigenvalues and s-Numbers. Cambridge University Press, New York (1986)

26. Raj, K., Sharma, S.K., Kumar, A.: Multiplication operator on Musielak-Orlicz spaces of Bochner type. J. Adv. Stud. Topol. 3, 1-7 (2012)

27. Sharma, A., Raj, K., Sharma, S.K.: Products of multiplication composition and differentiation operators from $H^{\infty}$ to weighted Bloch spaces. Indian J. Math. 54, 159-179 (2012)

28. Singh, R.K., Kumar, A.: Multiplication operators with closed range. Bull. Aust. Math. Soc. 16, 247-252 (1977)

29. Singh, R.K., Manhas, J.S.: Multiplication operators and composition operators with closed ranges. Bull. Aust. Math. Soc. $16,247-252(1977)$

30. Singh, R.K., Manhas, J.S.: Composition Operators on Function Spaces. North Holland, Amsterdam (1993)

31. Takagi, $H_{\text {., }}$ Yokouchi, K.: Multiplication and composition operators between two $L_{p}$-spaces. Contemp. Math. 232 321-338 (1999) 